\title{
Avaliação das propriedades físicas de revestimentos cerâmicos produzidos com resíduo da indústria cerâmica (cinza pesada de carvão mineral)
}

\author{
Physical properties evaluation of ceramic \\ tiles produced with the addition of ceramic \\ production residues - coal ash
}

Everton Skoronski ${ }^{1}$, Diego Hoefling Souza ${ }^{1}$, Samara dos Santos ${ }^{2}$,
Júlio Cesar Cesino $^{3}$, Marcos Gomes Ghislandi ${ }^{4}$

\begin{abstract}
${ }^{1}$ Departamento de Engenharia Ambiental, Universidade do Estado de Santa Catarina, Av. Luis de Camões 2090, Conta Dinheiro, 88520-000, Lages, SC, Brasil.

${ }^{2}$ Curso de Engenharia Química, Universidade do Sul de Santa Catarina, Av. José Acácio Moreira 797, Dehon, 88704-900, Tubarão, SC, Brasil.

${ }^{3}$ CECRISA, Unidade 02, R. S. João, s.n., S. João, 88708-450, Tubarão, SC, Brasil.

${ }^{4}$ Laboratory of Materials and Interface Chemistry, Eindhoven University of Technology, 5600 MB Eindhoven, The Netherlands

e-mail: skoronski@cav.udesc.br
\end{abstract}

\section{RESUMO}

A cinza resultante da queima do carvão mineral pulverizado é um dos resíduos gerados pelas empresas produtoras de materiais cerâmicas. Quimicamente, é constituída de uma ampla mistura de óxidos, sendo os majoritários $\mathrm{Al}_{2} \mathrm{O}_{3}, \mathrm{SiO}_{2}$ e $\mathrm{Fe}_{2} \mathrm{O}_{3}$ e outros menores como $\mathrm{CaO}, \mathrm{MgO}$, sulfatos e outras combinações. Atualmente, em muitas empresas, a cinza produzida é aterrada em áreas licenciadas por órgãos ambientais. Com o ritmo acelerado de produção de revestimentos cerâmicos e em virtude da sua limitada vida útil é necessário a utilização de outros locais, adequados, para deposição das cinzas, medida que provoca um gasto mensal elevado. Diante deste cenário, a adição de cinza à matéria-prima, além de diminuir o impacto ao meio-ambiente, pode propiciar uma redução geral de custos, tanto pelo barateamento da produção, como pela diminuição daquantidade de cinzas a serem aterradas. Desta forma, o objetivo deste trabalho foi avaliar as propriedades físicas de revestimentos cerâmicos produzidos com a adição de cinza pesada de carvão mineral. Para sua realização, corpos de prova foram confeccionados com adição de 1 a 30\% de cinza em base mássica. Os corpos de prova foram armazenados dentro de placas refratárias e queimados em forno túnel a uma temperatura de $1084^{\circ} \mathrm{C}$ em um ciclo de 29 horas. Foram analisados parâmetros físicos para a barbotina e corpo de prova pós-queima. Os resultados obtidos foram comparados com os padrões estabelecidos pela norma NBR 13818. Foi demonstrado que os experimentos realizados com adição de 1 a $10 \%$ de cinza apresentaram-se dentro dos padrões estipulados pela norma. Para porcentagens de cinza adicionada acima de $4 \%$, alguns valores estiveram fora dos padrões, sendo a formação de "coração negro" o parâmetro que mais se afastou das exigências da norma em questão.

Palavras-chave: cinza, revestimentos cerâmicos, propriedades físicas.

\section{ABSTRACT}

The ash is a solid waste generated in ceramic processing, being one of the outcomes of burning pulverized coal. The chemical composition of ash consists of a mixture of oxides, especially $\mathrm{Al}_{2} \mathrm{O}_{3}, \mathrm{SiO}_{2}$ and $\mathrm{Fe}_{2} \mathrm{O}_{3}$, and other species like calcium, magnesium, sulfates or other combinations. In many companies, the residual ash is transported to landfills, which are licensed by environmental agencies. With the increasing current production of ceramic tiles, the landfills have a limited lifetime, so the companies must search for other expensive alternatives for ash deposition. Henceforth, the addition of ash to the raw material, besides contribution to reduction of the environment impact, may also reduce the total costs, by decreasing the amount of raw material demanded in process and the amount of residual ash to be deposed. So, the objective of this study was to 
evaluate the physical properties of ceramic tiles produced with the addition of coal ash. Specimens were prepared by adding 1 to $30 \%$ of ash to mass base. The specimens were stored in refractory recipients and fired in a tunnel furnace at the temperature of $1084{ }^{\circ} \mathrm{C}$ in a 29 hours cycle. Physical parameters were analyzed for slip and specimens after firing. The results were compared with standards according to NBR 13818 . The results showed that properties of specimens produced with the addition of 1 up to $10 \%$ of ash are in accordance with NBR 13818. For samples with higher percentages of ash, e.g. above $4 \%$, some parameters did not achieve the norm standards. The formation of "black core" was the main problem for these samples.

Keywords: ash, ceramic tiles, physical properties.

\section{INTRODUÇÃO}

O mercado cerâmico brasileiro apresenta-se altamente pujante e competitivo em nível internacional. Segundo a Associação Nacional de Fabricantes de Cerâmica para Revestimento (ANFACER), o Brasil produziu, em 2011, cerca de 802,5 milhões de metros quadrados de cerâmica de revestimento, ficando atrás somente da China [1]. Alinhado a esta elevada produção, acompanha-se a geração de resíduos que, em uma empresa cerâmica, podem ser gerados em diversos setores, sendo os principais: restos de matéria-prima, resíduos de queima, gases tóxicos e produtos fora de especificação. Dentre estes resíduos, encontra-se a cinza pesada obtida da queima de carvão mineral em fornos cerâmicos para produção de revestimentos. Diversos estudos apontam a utilização de cinza para fabricação de novos materiais [2-6] , no entanto ainda existem poucos trabalhos apresentando a viabilidade de uso da cinza de carvão mineral produzida no próprio processo produtivo cerâmico.

A utilização de cinza pesada de carvão mineral no processo cerâmico apresenta diversas vantagens, tanto ambientais quanto econômicas, favorecendo a condução de processos industriais sustentáveis. Tais processos ainda não são utilizados no Brasil com a mesma intensidade que em países desenvolvidos [7]]. Atualmente as empresas depositam esse resíduo em terrenos licenciados por órgãos ambientais, mas estas áreas são limitadas. Outra alternativa de destinação adequada consiste na disposição em aterros industriais, uma vez que as cinzas pesadas são classificadas como um resíduo sólido de classe IIA (ABNT 10004), o que acaba onerando os custos de processamento nas empresas cerâmicas [ㅁ-10]. Com base neste cenário, a incorporação da cinza pesada na massa cerâmica utilizada para a fabricação de revestimentos cerâmicos apresenta-se como uma alternativa bastante atraente, uma vez que esta iniciativa confere uma destinação adequada ao resíduo em questão e minimiza os custos operacionais por substituir matéria-prima no processo.

Os minerais argilosos usados nas formulações de composições para revestimentos cerâmicos, frequentemente, contêm apreciáveis quantidades de substâncias capazes de reagir com o oxigênio, quando a temperatura é suficientemente elevada [11]. Estas substâncias são principalmente: carbono e matéria orgânica, enxofre e seus compostos, e alguns óxidos de metais de transição (principalmente ferro) quando em estados inferiores de valência (I), que podem gerar defeitos no produto cerâmico sinterizado. Quando a oxidação é incompleta, o produto cerâmico queimado apresenta alterações de cor e de textura em seu interior. Um núcleo escuro, conhecido como "coração negro", desenvolve-se durante a queima devido a presença de resíduos de matéria orgânica presentes na cinza. Sua origem é devida à resíduos de carbono formados por decomposição térmica da matéria orgânica contida nas argilas, mas a cor escura do centro é devida ao ferro que está presente em um estado reduzido. A principal fonte de redução do óxido férrico $\left(\mathrm{Fe}_{2} \mathrm{O}_{3}\right)$ para óxido ferroso $(\mathrm{FeO})$ é a presença de um resíduo carbonáceo resultante da carbonização de impurezas orgânicas [12].

O objetivo deste trabalho foi avaliar as propriedades físicas de revestimentos cerâmicos produzidos pela aplicação de cinza pesada de carvão mineral utilizada em uma indústria cerâmica do sul de Santa Catarina. Foram avaliadas as propriedades do material preparado com a adição de diferentes quantidades de resíduos à matéria-prima atualmente utilizada no processo de fabricação.

\section{MATERIAIS E MÉTODOS}

Para a realização deste trabalho uma barbotina foi preparada de acordo com a composição apresentada na Tabela I. A cinza utilizada foi obtida junto à Cecrisa Revestimentos Cerâmicos S/A - UN 02, situada em Tubarão/SC. Atualmente a empresa gera 125 toneladas mensais de cinza residual, que é produzida a partir da queima de carvão mineral e recuperada em ciclones instalados no sistema de purificação de gases da empresa.

A composição da cinza em termos de óxidos de alumínio, cálcio, ferro, magnésio, potássio, silício, sódio e titânio foi determinada através da técnica de fluorescência de raios X dispersiva, através de um equipamento de fluorescência de raios X de campo modelo X-MET 300 (Oxford Instruments). Foram adicionadas à formulação apresentada na Tabela 1 quantidades de cinza equivalentes a 1, 2, 3, 4, 5, 10, 15, 20 e 30\% 
em base mássica de material seco. Uma amostra isenta de adição de cinza foi utilizada como referência. Cada componente da formulação foi previamente seco em estufa por 4 horas a $105^{\circ} \mathrm{C}$, tendo seu tamanho de partícula posteriormente reduzido em moinho do tipo martelo. Após a secagem, as misturas foram submetidas à moagem por 23 minutos em moinho de bolas, com a adição de $63 \%$ de água e 1,3\% de defloculante. Uma amostra de $100 \mathrm{~mL}$ foi removida para verificação de densidade em picnômetro de $100 \mathrm{~cm}^{3}$, tempo de escoamento em copo Ford e teor de resíduo de moagem $\left(\mathrm{g} / \mathrm{g}_{\text {barbotina }}\right)$ em peneira Tyler325 mesh.

Tabela 1: Formulação utilizada para confecção da massa aplicada na obtenção dos corpos de prova.

\begin{tabular}{|c|c|}
\hline COMPONENTE & $\begin{array}{l}\text { COMPOSIÇÃO } \\
\left(\mathrm{g} / 100 \mathrm{~g}_{\text {matéria seca }}\right)\end{array}$ \\
\hline Argila A & 11,02 \\
\hline Argila B & 11,54 \\
\hline Caulim & 2,50 \\
\hline Argila C & 14,23 \\
\hline Resíduo A & 7,93 \\
\hline Resíduo B & 2,00 \\
\hline Calcário & 17,00 \\
\hline Talco & 10,00 \\
\hline Argila D & 7,50 \\
\hline Argila E & 14,62 \\
\hline Chamote & 1,66 \\
\hline
\end{tabular}

Resíduo A - biscoito cerâmico não conforme.

Resíduo B - revestimento cerâmico não conforme.

Para a confecção dos corpos de prova, a barbotina obtida foi submetida à secagem em estufa a $105^{\circ} \mathrm{C}$ por um período de $6 \mathrm{~h}$. A massa seca resultante foi novamente moída em um moinho de bolas por 6 min e em seguida separada em uma peneira com malha de abertura 20 mesh Tyler. Para conferir plasticidade à massa, água foi adicionada até ser atingido teor de umidade igual a $6 \%$. Uma amostra de $70 \mathrm{~g}$ foi coletada para confecção do corpo de prova. A massa foi adicionada a uma caixa matriz e submetida à compactação em uma

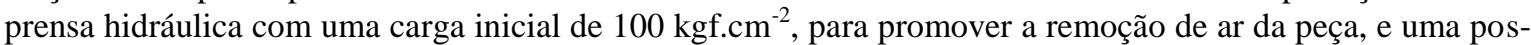
terior de $250 \mathrm{kgf.cm}$, para garantir uma boa compactação. Os corpos de prova verde foram secos em estufa a $105^{\circ} \mathrm{C}$ e, em seguida, queimados em forno túnel a $1073^{\circ} \mathrm{C}$. Este procedimento foi realizado para todas as formulações, com variação do teor de cinza aplicado.

Foram analisados os seguintes parâmetros físicos referentes aos corpos de prova: módulo de resistência à flexão (MRF), carga de ruptura (CRup), coeficiente de dilatação linear (CDL), absorção de água (AA) e presença de coração negro. Os métodos analíticos aplicados seguiram as recomendações da norma NBR 13818:1987. Todos os ensaios foram realizados em triplicata e as médias foram comparadas entre si com a aplicação do teste de Tukey a um nível de significância de 5\%.

\section{RESULTADOS E DISCUSSÃO}

A composição da cinza analisada através da técnica de fluorescência de raios X dispersiva apresentou a composição, em porcentagem mássica, ilustrada na Tabela 2

Tabela 2:Composição química da cinza utilizada para confecção dos corpos de prova.

\begin{tabular}{l|l|l|l}
\hline ELEMENTOS & $\%\left(\mathbf{g} / \mathbf{g}_{\text {MATÉRIA SECA }}\right)$ & ELEMENTOS & $\%\left(\mathbf{g} / \mathbf{g}_{\text {MATÉRIA SECA }}\right)$ \\
\hline Alumínio & 21,86 & Potássio & 2,87 \\
\hline Cálcio & 2,81 & Silício & 60,4 \\
\hline Ferro & 7,20 & Sódio & 1,22 \\
\hline
\end{tabular}


SKORONSKI, E.; SOUZA, D. H.; SANTOS, S. dos.; CESINO, J. C.; GHISLANDI, M. G. revista Matéria, v.20, n.1, pp. 239 - 244, 2015.

\begin{tabular}{l|l|l|l}
\hline Magnésio & 0,61 & Titânio & 0,27 \\
\hline
\end{tabular}

Os valores encontrados evidenciam a elevada presença de elementos que apresentam grande interesse para incorporação em materiais cerâmicos, principalmente silício e alumínio. Estes resultados motivaram o estudo da aplicação deste resíduo como substituinte de matérias-primas constituídas de óxidos, especialmente as argilas minerais.

A partir da determinação da composição da cinza, foi avaliada a influência da adição da cinza pesada nas propriedades físicas da barbotina. Os resultados encontram-se listados na Tabela 3. Observa-se uma diminuição na densidade da barbotina em função da adição de cinza pesada a este material. Para valores de teor de cinza adicionada acima de 5\%, a densidade apresenta valores menores do que $1,620 \mathrm{~g} . \mathrm{cm}^{-3}$. Valores baixos de densidade podem comprometer a estrutura mecânica do material cerâmico, devido à reduzida quantidade de óxidos presentes na estrutura. O tempo de escoamento, que está relacionado à viscosidade da barbotina, se apresentou dentro da faixa de referência (15 a 20 segundos) para todas as quantidades de cinza adicionadas, com exceção do teste envolvendo a adição de $30 \%$ de cinza, que resultou em um elevado tempo de 28,84 s. Com relação ao teor de resíduo de moagem, valores de cinza adicionada a barbotina acima de 5\% estão relacionados a um elevado valor de resíduo, acima de $6 \%$ (valor definido como crítico pela Cecrisa Revestimentos Cerâmicos S/A e grande parte das empresas do setor). A barbotina com elevado teor de resíduo ocasiona maiores desgastes nos equipamentos da produção, além de promover uma maior retração no processo de sinterização.

Tabela 3: Efeitos da adição da cinza pesada nas propriedades físicas da barbotina.

\begin{tabular}{l|l|l|l}
\hline $\begin{array}{l}\text { TEOR DE CINZA } \\
\text { ADICIONADA (\%) }\end{array}$ & $\begin{array}{l}\text { DENSIDADE } \\
\left(\mathbf{g} \cdot \mathbf{c m}^{-3}\right)\end{array}$ & $\begin{array}{l}\text { TEMPO DE ES- } \\
\text { COAMENTO (s) }\end{array}$ & RESIDUO (\%) \\
\hline Branco & $1,625 \mathrm{c}$ & $15,00 \mathrm{a}$ & $5,73 \mathrm{bc}$ \\
\hline 1 & $1,623 \mathrm{c}$ & $16,14 \mathrm{~b}$ & $5,10 \mathrm{a}$ \\
\hline 2 & $1,624 \mathrm{c}$ & $16,21 \mathrm{~b}$ & $5,95 \mathrm{c}$ \\
\hline 3 & $1,625 \mathrm{c}$ & $17,41 \mathrm{c}$ & $5,37 \mathrm{~b}$ \\
\hline 4 & $1,622 \mathrm{c}$ & $17,47 \mathrm{c}$ & $5,52 \mathrm{~b}$ \\
\hline 5 & $1,627 \mathrm{c}$ & $18,68 \mathrm{~d}$ & $5,63 \mathrm{~b}$ \\
\hline 10 & $1,578 \mathrm{a}$ & $19,94 \mathrm{ef}$ & $6,99 \mathrm{~d}$ \\
\hline 15 & $1,601 \mathrm{~b}$ & $21,20 \mathrm{f}$ & $7,00 \mathrm{~d}$ \\
\hline 30 & $1,581 \mathrm{a}$ & $23,61 \mathrm{~g}$ & $6,92 \mathrm{~d}$ \\
\hline Valores de referência* & $1,609 \mathrm{~b}$ & $28,84 \mathrm{~h}$ & $8,78 \mathrm{e}$ \\
\hline
\end{tabular}

As médias nas colunas seguidas pela mesma letra não diferem estatisticamente entre si ao nível de 5\% de probabilidade.*Valores de referência adotados internamente pela Cecrisa Revestimentos Cerâmicos S/A.

Após a determinação das características da barbotina, foram confeccionados corpos de prova, que foram submetidos ao processo de queima. Os corpos tiveram suas propriedades físicas determinadas e os valores obtidos encontram-se apresentados na Tabela 4.

Em termos do parâmetro CDL, observa-se que a adição de cinza influencia significativamente este valor, sendo que um maior incremento de cinza na barbotina tende a diminuir o CDL. Para uma adição de cinza de até $10 \%$, é possível obter material dentro das especificações da norma regulamentadora vigente. Peças cerâmicas com baixo CDL caracterizam um material com empeno positivo ou negativo. Com relação à MRF, observa-se que elevadas porcentagens de cinza incorporadas na barbotina, acima de $15 \%$, induzem a formação de um material com menor resistência à flexão. Para valores abaixo de $15 \%$ de cinza adicionada, os corpos de prova apresentaram resistência em conformidade aos limites mínimos propostos pela NBR 13818:1997. A absorção de água apresentou valores elevados, acima do máximo permitido pela regulamentação para os testes envolvendo adições maiores que $15 \%$ de cinza. Com relação à CRup, observa-se que a mesma apresentou-se acima do valor de referência $(200 \mathrm{~N})$ e que os testes realizados não apresentaram diferença significativa entre si. 
SKORONSKI, E.; SOUZA, D. H.; SANTOS, S. dos.; CESINO, J. C.; GHISLANDI, M. G. revista Matéria, v.20, n.1, pp. 239 - 244, 2015.

Tabela 4: Efeitos da adição de cinza pesada nas propriedades físicas do corpo de prova pós-queima. As médias seguidas pela mesma letra não diferem estatisticamente entre si ao nível de $5 \%$ de probabilidade.

\begin{tabular}{|c|c|c|c|c|}
\hline $\begin{array}{l}\text { TEOR DE CINZA } \\
\text { ADICIONADA (\%) }\end{array}$ & $\mathrm{CDL}\left(\mathrm{mm} \cdot{ }^{\circ} \mathrm{C}^{-1}\right)$ & CRup (N) & MRF (Kgf.cm-2) & AA (\%) \\
\hline Branco & $63.46 \mathrm{~cd}$ & $360,68 \mathrm{a}$ & $210,45 \mathrm{c}$ & $19,50 \mathrm{a}$ \\
\hline 1 & $64.06 \mathrm{~cd}$ & $368,05 \mathrm{a}$ & $215,59 \mathrm{c}$ & $19,77 \mathrm{a}$ \\
\hline 2 & $64.56 \mathrm{~d}$ & 409,33 a & $217,35 \mathrm{c}$ & 19,27 a \\
\hline 3 & $63.16 \mathrm{~cd}$ & $381,54 \mathrm{a}$ & $220,49 \mathrm{c}$ & $19,29 \mathrm{a}$ \\
\hline 4 & $63.03 \mathrm{~cd}$ & $400,87 \mathrm{a}$ & $213,37 \mathrm{c}$ & $19,62 \mathrm{a}$ \\
\hline 5 & $63.03 \mathrm{~cd}$ & $392,88 \mathrm{a}$ & $217,79 \mathrm{c}$ & $19,89 \mathrm{ab}$ \\
\hline 10 & $64.40 \mathrm{~d}$ & $403,36 \mathrm{a}$ & $214,25 \mathrm{c}$ & $19,97 \mathrm{ab}$ \\
\hline 15 & $58.00 \mathrm{~b}$ & $371,31 \mathrm{a}$ & $199,10 \mathrm{~b}$ & $20,06 \mathrm{~b}$ \\
\hline 20 & $58.06 \mathrm{~b}$ & $384,01 \mathrm{a}$ & $192,58 \mathrm{~b}$ & $21,02 \mathrm{c}$ \\
\hline 30 & $55.90 \mathrm{a}$ & $360,18 \mathrm{a}$ & $173,65 \mathrm{a}$ & $21,73 \mathrm{c}$ \\
\hline Valores de referência* & 63 a 67 & $>200$ & $>200$ & $<20$ \\
\hline
\end{tabular}

*Valor de referência conforme NBR 13818:1997 [13].

Com base nas propriedades físicas, verifica-se que é possível incorporar até $10 \%$ de cinza pesada da queima do carvão na barbotina, para fabricação de revestimentos cerâmicos, sem comprometer as propriedades do material. Entretanto, por inspeção visual, foi observada a presença de coração negro em intensidade acentuada para os testes envolvendo adição de cinza superior a 4\%. O coração negro pode ser visualizado com uma região escura (Figura 1) que se estende paralelamente à superfície, próxima a meia altura da espes-

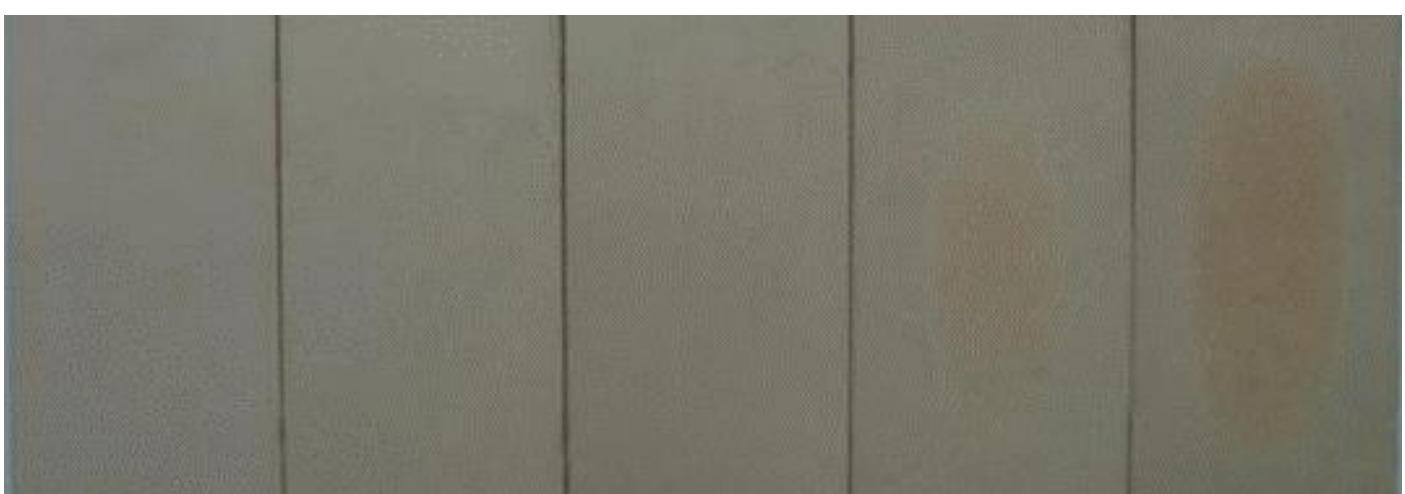

sura e ao longo de toda a peça. A região escura geralmente desaparece nas proximidades das bordas da peça porque nas extremidades normalmente ocorre a desgaseificação. A presença do coração negro traz impactos visuais negativos para a estética do revestimento cerâmico e pode ser um fator para classificar determinada peça como material não conforme $[\underline{14}, \underline{15}]$.

Figura 1: Imagem dos corpos de prova pós-queima. Da esquerda para a direita a adição de cinza foi igual a 1, 2, 3, 4 e $5 \%$.

\section{CONCLUSÕES}

Com base nos resultados apresentados é possível concluir que a adição de cinza pesada, obtida da queima de carvão mineral, na fabricação de revestimentos cerâmicos pode ser uma importante alternativa para a gestão 
SKORONSKI, E.; SOUZA, D. H.; SANTOS, S. dos.; CESINO, J. C.; GHISLANDI, M. G. revista Matéria, v.20, n.1, pp. 239 - 244, 2015.

ambiental em uma empresa cerâmica, sobretudo sob o ponto de vista de sustentabilidade e valorização de resíduos industriais. Neste trabalho observou-se que a adição de cinza pode atingir valores de até $10 \%$ em massa, sem que haja comprometimento das propriedades físicas do material, como densidade e tempo de escoamento. A inserção de até $4 \%$ em cinzas não gera a formação de "coração negro", defeito que pode comprometer a qualidade visual do revestimento. Desta forma, testes em larga escala devem ser realizados para avaliação das implicações em termos de processo industrial relacionados à substituição de matéria-prima tradicional por cinza, buscando estabelecer um eventual redesenho da unidade em busca do aproveitamento deste resíduo.

\section{AGRADECIMENTOS}

Os autores agradecem à CECRISA Revestimentos Cerâmicos S/A pelo suporte financeiro e à UNISUL pela realização das análises de fluorescência de raios-X.

\section{BIBLIOGRAFIA}

[1] ASSOCIAÇÃO NACIONAL DE FABRICANTES DE CERÂMICA PARA REVESTIMENTO, http://www.anfacer.org.br/. Acessado em setembro de 2004.

[2] LIMA, S. A., SALES, A., ALMEIDA, F. C. R., et al., "Concretos com cinza do bagaço da cana-deaçúcar: avaliação da durabilidade e potencialidade de aplicação na construção civil”, Ambiente Construído (Online), v. 11, pp. 201-212, 2011.

[3] BEZERRA, I. M. T., SOUZA, J., QUEIROZ DE CARVALHO, J. B., et al.,“Aplicação da cinza da casca do arroz em argamassas de assentamento", Revista Brasileira de Engenharia Agrícola e Ambiental (Online), v. 15, pp. 639-645, 2011.

[4] MEDEIROS, E. N. M., SPOSTO, R. M., NEVES,G., et al.,"Incorporação de cinza de lenha, lodo de estação de tratamento de água e cinza de casca de arroz em massa cerâmica. Utilização da técnica de planejamento", Cerâmica, v. 56, pp. 399-404, 2010.

[5] RODRIGUES, M. S., BERALDO, A. L.,"Caracterização física e mecânica de argamassas à base de cimento Portland e cinza de casca de arroz residual”, Engenharia Agrícola, v. 30, pp. 193-204, 2010.

[6] DELLA, V. P., HOTZA, D., KUHN, I., "Caracterização de cinza de casca de arroz para uso como matéria-prima na fabricação de refratários de sílica”,Química Nova (Impresso), v. 24, n.6, pp. 778-782, 2001.

[7] LEROY, C., FERRO, M. C., MONTEIRO, R. C. C., et al.,"Production of glass-ceramics from coal ashes", Journal Of The European Ceramic Society, v. 21, n. 2, pp. 195-202, 2001.

[8] KARANGELOS, D. J., ROUNI, P. K., PETROPOULOS, N. P., et al.,"Radioenvironmental survey of the Megalopolis power plants fly-ash deposits", Radioactivity In The Environment, v.7, pp. 1025-1029, 2005.

[9] FUNGARO, D. A., BRUNO, M.,"Remoção de azul de metileno de solução aquosa usando zeólitas sintetizadas com amostras de cinzas de carvão diferentes”,Química Nova (Impresso), v. 32, pp. 955-959, 2009.

[10] SILVA, M. L., LANGE, L. C.,"Caracterização das cinzas de incineração de resíduos industriais e de serviços de saúde”,Química Nova, v. 31, pp. 199-203, 2008.

[11] BARBA, A., MORENO, A., NEGRE, F., et al., "Oxidation of black cores in firing”, TBI, v. 6, n. 5, pp.17-23, 1990.

[12] KINGERY, W. D., BOWEN, H. K., UHLMANN, D. R.,Introduction to ceramics,2 ed., New York, John Wiley \& Sons, 1976.

[13] ASSOCIAÇÃO BRASILEIRA DE NORMAS TÉCNICAS,NBR 13818: Versão Corrigida. Placas Cerâmicas para revestimento - especificação e métodos de ensaios, Rio De Janeiro, ABNT, 1997.

[14] SANTOS, I. M. G. dos, SILVA, J. M., TRINDADE, M. F. S., et al.,"Efeito da Adição de Rejeito na Redução de Coração Negro em Cerâmicas Vermelhas” Cerâmica, São Paulo, Brasil, v. 51, n.318, pp. 144150,2005

[15] MELCHIADES, F. G., DAMIANI, J. C., PEREZ, F., et al.,"Coração negro em revestimentos cerâmicos: Principais causas e possíveis soluções”, Cerâmica Industrial, v. 6, n.2, pp. 12-16, 2001. 